\title{
Safety and feasibility of biventricular devices reuse in general and elderly population - a single-center retrospective cohort study
}

This article was published in the following Dove Press journal:

Clinical Interventions in Aging

12 August 2015

Number of times this article has been viewed

\author{
Raluca Șoșdean ${ }^{1,2}$ \\ Cristian Mornoș 1,2 \\ Bogdan Enache ${ }^{1,2}$ \\ Răzvan I Macarie ${ }^{2}$ \\ Raluca lanoș ${ }^{2}$ \\ Ana-Maria Ștefea ${ }^{2}$ \\ Sorin Pescariu' ${ }^{1,2}$ \\ 'Department of Cardiology, "Victor \\ Babeș" University of Medicine and \\ Pharmacy, ${ }^{2}$ Cardiology Clinic, Institute \\ of Cardiovascular Medicine, Timișoara, \\ Romania
}

Introduction: Cardiac resynchronization therapy (CRT) is known to have very important beneficial effects on heart failure patients. Unfortunately, biventricular implantable cardiac devices (CRT devices), through which this therapy is implemented, are very expensive and sometimes hard to achieve, especially in underdeveloped/developing economies, making this an important problem of public health. As a possible solution, CRT reuse is of great interest nowadays, but unlike simple devices, data in the literature are scarce about biventricular device reuse.

Aim: To address safety concerns, we aimed to analyze infection burden in the general and elderly population and also early battery depletion and generator malfunction of resterilized biventricular devices compared to new devices.

Methods: A cohort of 261 CRT patients (286 devices), who underwent implantation between 2000 and 2014, was retrospectively analyzed. The study group included 115 patients and 127 resterilized devices, that was divided into a subgroup of 69 elderly patients ( $\geq 60$ years) and 74 devices and a subgroup of 47 younger patients ( $<60$ years) and 53 devices, and the control group included 146 patients and 159 new devices. The groups were compared using a multivariate logistic regression model.

Results: A number of 12 (4.2\%) infectious complications were encountered, five (3.9\%) in the study group and seven (4.4\%) in the control group (odds ratio, 2.83 [0.59-13.44], $P=0.189$ ), one $(1.3 \%)$ in the elderly and four (7.5\%) in the younger subgroup (odds ratio, 3.80 [0.36-40.30], $P=0.266$ ), with no statistically significant difference between them. There was only one case of early battery depletion, after 17 months, in one study group patient. No generator malfunction was detected.

Conclusion: Reuse of biventricular cardiac implantable electronics seems feasible and safe in both the general population and the elderly population, and it could be a promising alternative when new devices cannot be obtained in a safe period of time.

Keywords: biventricular implantable cardiac devices, reuse, infectious complications, malfunction

\section{Introduction}

Cardiac resynchronization therapy (CRT), implemented through biventricular cardiac implantable electronic devices (CIEDs), is a relatively new and revolutionary therapy for heart failure patients resistant to maximal medical therapy. It is proven to regress the left ventricle's pathological remodeling, reduce the degree of mitral regurgitation if present, increase the left ventricle's ejection fraction, ameliorate the patient's clinical status, increase the quality of life, and decrease heart failure hospitalization rate as well as the mortality. ${ }^{1}$ The most representative clinical trials showed this therapy to reduce all-cause mortality by $28 \%$ and new heart failure hospitalizations by $37 \%$ for these
Correspondence: Sorin Pescariu Cardiology Clinic, Institute of Cardiovascular Medicine, I3A, Gheorghe Adam Street, Timișoara 300310, Romania Tel +40256 207355

Fax +40 256207362

Email sorinpescariu@yahoo.com 
patients. ${ }^{1}$ The median life expectancy for patients treated with medical therapy and CRT is 8 years compared to 3.5 years for patients treated in the past with medical therapy alone. ${ }^{2}$ Unfortunately, this therapy is also very expensive, and this issue restricts its availability and deprives a significant number of patients of its beneficial and often life-saving effects, especially in developing economies. Impossibility to provide medical care in certain conditions is one of the most important and debated problems of public health nowadays. ${ }^{3}$

A number of studies described simple single-chamber and dual-chamber pacemaker reuse as a safe practice, but future studies and time are needed for it to gain general confirmation and acceptance..$^{3-5}$ Regarding biventricular devices reuse, through which CRT is implemented, data in the literature are scarce. Unlike simple devices, these require a significantly longer procedural time for their implantation, especially because of the difficulties the electrophysiologist may encounter while inserting the coronary sinus lead, besides the higher number of leads. ${ }^{6}$ Biventricular devices were proved to be an independent risk factor for device infection. ${ }^{4}$ They are implanted in patients with impaired left ventricle function and advanced heart failure, thus with an impaired immunity (poor defense capacity), and if possible, they are set to work more than $98 \%$ of the time in order to properly synchronize the heart. Because of these particularities, it may be questionable if their behavior is not different regarding infectious complications (several cumulated predisposing factors) and device functionality, when being reused. Also, it has been proposed that the resterilized devices are better suited to be implanted in older patients, as the need for repeated replacement would be lower. ${ }^{7}$ In some studies, patients who received reused single- and/or dual-chamber devices were older than the ones receiving new ones, ${ }^{8}$ and still had no significant difference in the infection burden. In other studies, age was found to predict device infection. ${ }^{4,9}$ After a detailed search of the literature, we found no data for reused biventricular devices regarding this issue. Unlike simple CIEDs, these devices might have a different behavior when associating advanced age and other comorbidities that come along with them to the high number of leads and the difficult implantation intervention.

We aim to analyze in this study the feasibility and safety of biventricular devices' reuse, setting as a combined primary end-point the infection burden, early battery depletion, and device (generator) malfunction. Secondary, we proposed to analyze the infection burden accompanying reused devices in the elderly population compared to the younger population in order to verify whether supplemental safety measures should be adopted in this population.

\section{Methods}

A single-center cohort of patients consecutively implanted with biventricular devices (pacemakers and/or defibrillators) from 2000 to 2014 at the Institute of Cardiovascular Diseases from Timişoara, Romania, was retrospectively included in the study. Device replacement interventions for battery depletion and upgrade interventions were also included. Some patients had only the second implantation performed in our clinic. Patients who had second implantation after an infection as well as those who were early lost to follow-up were excluded. The patients with biventricular devices were divided into two groups: the study group, including the resterilized devices, that was further divided into a subgroup of patients under 60 years of age and a subgroup of patients over 60 years of age, and the control group including the new devices. The CRT devices were implanted after a thorough evaluation by an electrophysiology expert, according to the indications of the current European Society of Cardiology guidelines for pacing and CRT from 2013, ${ }^{2} 2010$ (update), ${ }^{10}$ and 2007, ${ }^{11} 2002$ American College of Cardiology/American Heart Association/North American Society of Pacing and Electrophysiology implant guideline update, ${ }^{12}$ and representative studies in this domain and the protocols established in the clinic. Resterilized devices were implanted only when new devices were unavailable in the hospital, and the patients could not receive a new one within a reasonable amount of time, estimated by their physician after analyzing their cardiac status. The patients underwent the implant intervention only after signing an informed consent included in the clinic's patient files. Besides the benefits, they were all precisely informed about the implantation technique and especially about the complications that could arise during and after the intervention, specifically for resterilized devices.

The refurbished devices were received from "Stimubanque" (Nancy, France), an institution specialized in legally collecting these devices mostly from hospital donations in order to further donate them to hospitals from countries that might benefit. The devices were explanted postmortem or during an upgrade intervention, but also devices that were accidentally desterilized or were improperly sealed were included. The devices were carefully inspected for external damage and properly interrogated by an electrophysiologist, before and after sterilization and also before implantation. Only the devices with a perfect external integrity, good functioning parameters, and a minimum expected battery life of 3 years were considered for reuse. Part of these devices was sterilized in France, and the other part, in Romania, in our hospital.

The standard preparation and sterilization of the devices consisted first in fine brushing with water and soap. Then, 
they were kept in a phenoxypropanol and benzalkonium chloride solution for 24 hours, washed with water, and air-dried for 24 hours. They were washed again with $70 \%$ ethanol, and finally, packed in a special bag, sealed, and sterilized with ethylene oxide for 24 hours. All bags were labeled with the sterilization date, in order to repeat the procedure if a standard period of 3 months is exceeded before device reuse.

The devices were implanted using subclavian vein puncture alone, cephalic vein cut-down alone, or a double venous approach - cephalic and subclavian. The last-mentioned technique was frequently used in our hospital in the past few years in order to reduce the possible risk of lead damage, vein thrombosis, and infection, when a high number of leads are inserted through a single approach, ${ }^{13}$ especially through a smaller vein like the cephalic. It is also used to reduce the risk of pneumothorax when puncturing the subclavian vein, as a guidewire is first introduced through the cephalic vein to serve as a radiologic marker for the puncture. All patients, receiving a new or a resterilized device, received three doses of antibiotic (usually amoxicillin with clavulanate), one before the intervention and two after, at a time distance of 12 hours between doses. The implantation wound was examined, and new dressing was applied every 2 days until suture removal (after 7 days for pacemakers and after 10 days for defibrillators). The control visits were established at 1 month, 3 months, and two times per year thereafter.

Patient data regarding age, sex, the etiology of the dilated cardiomyopathy, New York Heart Association class, conduction delay, and other factors that might predispose to infection (diabetes, hypertension, chronic kidney disease, obesity, atrial fibrillation, chronic steroid use $)^{3}$ were obtained from the patient files stored in the hospital's archive. Data about the devices, concerning their type (pacemaker/defibrillator, two/three leads), implantation technique (as it might influence the infection burden) and type (first implantation, second implantation - upgrade, or device change for battery depletion), acute pacing and sensing thresholds, and complications needing reintervention, were obtained from the intervention registries stored in the hospital's electrophysiology laboratory archive.

The data were than statistically processed and analyzed in order to obtain the results according to the established end-points: device-related infection - concerning the pocket and/or leads, early battery depletion - considered at less than 2 years (24 months), and device malfunction requiring reintervention. Our institutions Ethics Committee, in accordance with national law on human subject research, has exempted our study from Institutional Review Board approval as our single-center study involves exclusively pre-existing anonymous data.

\section{Statistical analysis}

The statistical analysis was performed by using STATA version 12.0 (STATACorp, College Station, TX, USA). Patient/ device characteristics are expressed as mean \pm 1 standard deviation or as a number and percentage, as appropriate. Time variables are expressed as median value with interquartile $1(0.25 \%)$ and $3(0.75 \%)$ interval. Numerical and qualitative variables were compared using Student's $t$-test and chi square test, respectively. Variables which were found to be significantly different between the two groups/subgroups were included in a multivariable logistic regression analysis. We used a multivariate logistic regression model in order to test the null hypothesis that there is no difference in the analyzed values between the groups. Covariables were used to control for confounders and effect modification. The model was adjusted for the presence of chronic kidney disease, presence of atrial fibrillation, QRS length, and device type when comparing the two groups and for chronic kidney disease and device type when comparing the subgroups. We considered a $P$-value of $<0.05$ as statistically significant.

\section{Results}

There were a total number of 261 patients receiving 286 devices: a number of 115 patients in the study group, who received 127 resterilized devices, and a number of 146 patients in the control group, who received 159 new devices. The groups were similar considering patient characteristics and device and intervention-related aspects, except for chronic kidney disease, atrial fibrillation, QRS width, and defibrillators number, which were higher in the reused group - study group. In the study group, the subgroup of patients over 60 years of age included 69 patients who received 74 resterilized devices, and the subgroup under 60 years of age included 47 patients who received 53 resterilized devices. One of the patients in the study group was implanted at 58 years (fitting in the young patients subgroup) and had it replaced at 61 years (thus fitting in the elderly patients subgroup). Besides the age, chronic kidney disease was higher in the elderly subgroup, as it was expected. Device type and lead number were different, favoring the elderly subgroup. All these parameters are summarized in Tables 1-3.

The median follow-up was of 35 months (interquartile range, 17-57 months, with a maximum follow-up of 94 months and a minimum follow-up of 4 months because of end-point achievement). 
Table I Patient characteristics at the moment of intervention, study group versus control group

\begin{tabular}{|c|c|c|c|}
\hline & Study group & Control group & $P$-value \\
\hline & I I 5 patients// 27 interventions & I46 patients/ I 59 interventions & \\
\hline Age, mean \pm SD (years) & $61.69 \pm 10.09$ & $61.03 \pm 9.40$ & $0.57 \mid$ \\
\hline Sex, n (\%) & & & 0.859 \\
\hline Male & $19(14.96 \%)$ & 25 (15.72\%) & \\
\hline Female & 108 (85.03\%) & I 34 (84.27\%) & \\
\hline Etiology of DCM, n (\%) & & & 0.062 \\
\hline Idiopathic & 76 (59.84\%) & $104(65.40 \%)$ & \\
\hline Ischemic & $36(28.34 \%)$ & 27 (16.98\%) & \\
\hline Valvular & 7 (5.5I\%) & $9(5.66 \%)$ & \\
\hline Myocarditis & I (0.78\%) & $0(0 \%)$ & \\
\hline Other & 7 (5.5 I\%) & 19 (11.94\%) & \\
\hline NYHA class, n (\%) & & & 0.649 \\
\hline Class II & $21(16.53 \%)$ & 31 (19.49\%) & \\
\hline Class III & $83(65.35 \%)$ & 95 (59.74\%) & \\
\hline Class IV & $23(18.11 \%)$ & $32(20.12 \%)$ & \\
\hline Arterial hypertension, $\mathrm{n}(\%)$ & $50(39.37 \%)$ & $73(45.91 \%)$ & 0.267 \\
\hline Diabetes mellitus, n (\%) & $31(24.40 \%)$ & $30(18.86 \%)$ & 0.256 \\
\hline Chronic kidney disease, n (\%) & $78(6|.4| \%)$ & $73(45.91 \%)$ & 0.009 \\
\hline Obesity, n (\%) & 31 (24.40\%) & $46(28.93 \%)$ & 0.392 \\
\hline Atrial fibrillation, n (\%) & 60 (47.24\%) & 49 (30.8I\%) & 0.004 \\
\hline Conduction delay, n (\%) & & & 0.630 \\
\hline LBBB & 96 (75.59\%) & 119 (78.84\%) & \\
\hline RBBB & 8 (6.29\%) & $7(4.40 \%)$ & \\
\hline QRS width, mean \pm SD (ms) & $160.28 \pm 33.34$ & $151.25 \pm 30.56$ & 0.029 \\
\hline Chronic steroid use, n (\%) & $0(0 \%)$ & $0(0 \%)$ & \\
\hline
\end{tabular}

Abbreviations: SD, standard deviation; $n$, number; DCM, dilated cardiomyopathy; NYHA, New York Heart Association; LBBB, left bundle branch block; RBBB, right bundle branch block; RV, right ventricle; CS, coronary sinus; RA, right atrium.

Table 2 Device and intervention-related aspects, study group versus control group

\begin{tabular}{|c|c|c|c|}
\hline & Study group & Control group & $P$-value \\
\hline & 127 devices & 159 devices & \\
\hline \multicolumn{3}{|l|}{ Device type, n (\%) } & 0.000 \\
\hline Pacemaker & $80(63 \%)$ & 143 (89.94\%) & \\
\hline Defibrillator & 47 (37\%) & $16(10.06 \%)$ & \\
\hline \multicolumn{3}{|l|}{ Lead number, n (\%) } & 0.404 \\
\hline Two, RV and CS & $63(49.60 \%)$ & 71 (44.65\%) & \\
\hline Three, RV, CS, and RA & $64(50.39 \%)$ & $88(55.34 \%)$ & \\
\hline \multicolumn{3}{|l|}{ Implantation technique, n (\%) } & 0.088 \\
\hline Cephalic vein & 48 (37.79\%) & $46(28.93 \%)$ & \\
\hline Subclavian vein & $36(28.34 \%)$ & $39(24.52 \%)$ & \\
\hline Double approach & $43(33.85 \%)$ & 74 (46.54\%) & \\
\hline \multicolumn{4}{|c|}{ Acute pacing threshold, mean $\pm S D(V)$} \\
\hline RA & $0.82 \pm 0.29$ & $0.93 \pm 0.40$ & 0.163 \\
\hline RV & $0.59 \pm 0.29$ & $0.57 \pm 0.18$ & 0.528 \\
\hline CS & $1.94 \pm 1.56$ & $2.0 \pm 1.31$ & 0.739 \\
\hline \multicolumn{4}{|c|}{ Acute sensing threshold, mean $\pm S D(m V)$} \\
\hline RA & $4.32 \pm 2.23$ & $4.46 \pm 3.16$ & 0.764 \\
\hline RV & $13.98 \pm 5.49$ & $14.06 \pm 5.22$ & 0.913 \\
\hline CS & $12.92 \pm 5.87$ & $13.92 \pm 5.92$ & 0.214 \\
\hline Replaced device, n (\%) & 34 (26.77\%) & $33(20.75 \%)$ & 0.233 \\
\hline Upgraded & 20 (15.74\%) & $17(10.69 \%)$ & 0.206 \\
\hline
\end{tabular}

Abbreviations: $n$, number; $R V$, right ventricle; $C S$, coronary sinus; $R A$, right atrium; $\mathrm{SD}$, standard deviation.
Thirty-four devices (in 32 patients) had to be explanted for battery depletion, 21 in the study group and 13 in the control group, after a median period of time of 40 months (interquartile range, 36-66 months) for the study group and 67 months (interquartile range, 56-74 months) for the control group $(P=0.01)$. Six patients/devices from the study group and seven patients/devices from the control group reached one of the primary outcomes in a similar median period of time of 13 months (interquartile range, 10-16 months) and 7 months (interquartile range, 5-8 months), respectively $(P=0.22)$.

We encountered a total number of $12(4.2 \%)$ infections in 12 different patients, similar between the two groups - five in the study group and seven in the control group $(P=0.84)$. The difference remained insignificant after adjusting for the significantly different variables between the groups - chronic kidney disease, atrial fibrillation, QRS width, and device type (presence of defibrillator function) ( $P=0.189$, odds ratio $=2.83,95 \%$ confidence interval: $0.59-13.44$ ). All variables had an insignificant impact, except for the device type (defibrillator function) (Table 4). All infections were related to the device pocket without signs of lead involvement and/or 
Table 3 Patient and device characteristics at the moment of intervention, elderly subgroup versus younger subgroup

\begin{tabular}{|c|c|c|c|}
\hline & Elderly subgroup & Younger subgroup & $P$-value \\
\hline & 69 patients/74 interventions & 47 patients/53 interventions & \\
\hline Age, mean $\pm S D$ (years) & $68.6 \pm 5.96$ & $52.03 \pm 5.81$ & \\
\hline Sex, n (\%) & & & 0.330 \\
\hline Male & $6 \mathrm{I}(82.43 \%)$ & 47 (I5.72\%) & \\
\hline Female & $13(17.56 \%)$ & $6(84.27 \%)$ & \\
\hline Etiology of DCM, n (\%) & & & 0.681 \\
\hline Idiopathic & $46(62.16 \%)$ & $30(56.60 \%)$ & \\
\hline Ischemic & $21(28.37 \%)$ & $15(28.30 \%)$ & \\
\hline Valvular & $4(5.4 \%)$ & $3(5.66 \%)$ & \\
\hline Myocarditis & $0(0 \%)$ & I (I.88\%) & \\
\hline Other & $3(4.05 \%)$ & $4(7.54 \%)$ & \\
\hline NYHA class, n (\%) & & & 0.617 \\
\hline Class II & 14 (|8.9|\%) & $7(\mid 3.20 \%)$ & \\
\hline Class III & 48 (64.86\%) & $35(66.03 \%)$ & \\
\hline Class IV & $12(16.21 \%)$ & II (20.75\%) & \\
\hline Arterial hypertension, $\mathrm{n}(\%)$ & 32 (43.24\%) & $18(33.96 \%)$ & 0.291 \\
\hline Diabetes mellitus, n (\%) & $20(27.02 \%)$ & II (20.75\%) & 0.417 \\
\hline Chronic kidney disease, $\mathrm{n}(\%)$ & $53(71.62 \%)$ & $25(47.16 \%)$ & 0.005 \\
\hline Obesity, n (\%) & $18(24.32 \%)$ & $13(24.52 \%)$ & 0.979 \\
\hline Atrial fibrillation, $\mathrm{n}(\%)$ & $38(51.35 \%)$ & $22(41.50 \%)$ & 0.27 \\
\hline Conduction delay, $\mathrm{n}(\%)$ & & & 0.628 \\
\hline LBBB & 58 (78.37\%) & 38 (7I.69\%) & \\
\hline RBBB & $4(5.4 \%)$ & $4(7.54 \%)$ & \\
\hline $\mathrm{QRS}$ width, mean $\pm \mathrm{SD}(\mathrm{ms})$ & $164.75 \pm 32.17$ & $154.34 \pm 34.29$ & 0.110 \\
\hline Device type, n (\%) & & & 0.006 \\
\hline Pacemaker & $54(72.94 \%)$ & $20(37.73 \%)$ & \\
\hline Defibrillator & $20(27.02 \%)$ & $27(50.94 \%)$ & \\
\hline Lead number, n (\%) & & & 0.009 \\
\hline Two, RV and CS & $44(59.45 \%)$ & $19(35.84 \%)$ & \\
\hline Three, RV, CS, and RA & 30 (40.54\%) & $34(45.94 \%)$ & \\
\hline Implantation technique, n (\%) & & & 0.100 \\
\hline Cephalic vein & 30 (40.54\%) & $18(52.83 \%)$ & \\
\hline Subclavian vein & $25(33.78 \%)$ & $12(22.64 \%)$ & \\
\hline Double approach & $19(25.67 \%)$ & $23(43.39 \%)$ & \\
\hline Replaced device, $n$ (\%) & $23(31.08 \%)$ & II (20.75\%) & 0.195 \\
\hline Upgraded & 14 (18.91\%) & $6(11.32 \%)$ & 0.249 \\
\hline
\end{tabular}

Abbreviations: SD, standard deviation; n, number; DCM, dilated cardiomyopathy; NYHA, New York Heart Association; LBBB, left bundle branch block; RBBB, right bundle branch block; RV, right ventricle; CS, coronary sinus; RA, right atrium.

endocarditis. Four of these patients had device exteriorization, without proven infection (three in the control group and one in the study group, eg, decubitus lesions), but the wound was considered and treated like an infection according to the current protocols. Seven patients had device exteriorization

Table 4 Logistic regression model of resterilized devices group versus new devices group

\begin{tabular}{lll}
\hline Variable & $\begin{array}{l}\text { Logistic regression } \\
\text { P-value }\end{array}$ & $\begin{array}{l}\text { Adjusted OR } \\
\text { (95\% Cl) }\end{array}$ \\
\hline Chronic kidney disease & 0.331 & $0.5 \mathrm{I}(0.13-1.96)$ \\
Atrial fibrillation & 0.120 & $2.9(0.75-1 \mathrm{I} . \mathrm{II})$ \\
QRS length & $0.26 \mathrm{I}$ & $1.0 \mathrm{I}(0.99-\mathrm{I} .03)$ \\
Device type & $0.02 \mathrm{I}$ & $5.75(\mathrm{I} .30-25.49)$ \\
\hline
\end{tabular}

Abbreviations: $\mathrm{OR}$, odds ratio; $\mathrm{Cl}$, confidence interval. with proven infection (three in the control group and four in the study group), and one patient in the study group had a chronic collection underneath the device, without external and/or systemic signs of infection. The collection was encountered during the device replacement intervention for battery depletion, 5.9 years after the first implant intervention. Because of this long period of time, it might be questionable whether the infection was linked to the first intervention, but given the site of the collection (deep into the pocket, under the device), without any other intervention in this area, with inoculation potential, we considered it as a chronic occult infection identifiable as a primary end-point. Besides this particular exception, we found that this outcome occurred in the first 2 years after implantation. All device exteriorizations 
with proven infections occurred within the 1st year after implantation along with two device exteriorizations without proven infection. The remaining two exteriorizations without proven infection (one in the study group and one in the control group) occurred between the 1 st and the 2 nd year after implantation.

Regarding the two subgroups of the study group, we encountered a similar number of infectious complications: one $(1.3 \%)$ in the elderly subgroup and four $(7.5 \%)$ in the younger group $(P=0.07)$. The results were consistent after adjusting for device type and chronic kidney disease $(P=0.266$, odds ratio $=3.80,95 \%$ confidence interval: 0.36-40.30). Neither variable was a significant predictor of infectious complications (Table 5). All infections involved three lead devices.

Regarding early battery depletion, one patient in the study group had to have his triple-chamber defibrillator replaced after 17 months ( 1 year and 5 months, second replacement), without any change in the device's parameters and without any shock delivery that might have led to an increase in battery consumption. There was no early battery depletion in the control group.

There were nine cases of pacing malfunction, all due to lead displacement without electronic device/generator involvement. We encountered six cases of coronary sinus lead displacement and three cases of right ventricle lead displacement, along with lead fracture in one of them.

\section{Discussion}

Our results show that biventricular CIEDs reuse is as safe as implantation of new devices in terms of infection burden, early battery depletion, and electronic system malfunction. The results show no worsening in the infection outcome for the elderly patients with resterilized devices included in our study, suggesting that there is no need for additional safety measures and that biventricular CIEDs can be safely reused also in this population in order to improve their health when appropriate.

We expected the period of time to device replacement for battery depletion to be shorter for the study group as

Table 5 Logistic regression model of elderly patients subgroup versus young patients subgroup

\begin{tabular}{lll}
\hline Variable & $\begin{array}{l}\text { Logistic regression } \\
\text { P-value }\end{array}$ & $\begin{array}{l}\text { Adjusted OR } \\
(\mathbf{9 5 \%} \mathbf{C l})\end{array}$ \\
\hline Chronic kidney disease & 0.801 & $0.77(0.10-5.57)$ \\
Device type & 0.161 & $5.17(0.52-51.39)$ \\
\hline
\end{tabular}

Abbreviations: $\mathrm{OR}$, odds ratio; $\mathrm{Cl}$, confidence interval. most of them have already functioned for a certain period of time. On the other hand, the resterilized devices showed a reasonable functioning time/battery life, suggesting a proper device selection before reuse to be feasible and useful. The infectious complications were encountered after similar periods of time, suggesting a similar response of the organism to reused devices. Previous studies established a number of risk factors for CIED infection: high number of leads (especially higher than two), impaired left ventricle function, chronic kidney disease, lack of antibiotic prophylaxis, chronic steroid use, and reinterventions. ${ }^{4,14}$ A retrospective study on 3,105 CIEDs implanted between 2000 and 2007 emphasized the importance of sterility, implantation technique, and antibiotic prophylaxis for heart failure patients implanted with biventricular devices, as they found the implantation of these devices to be an independent predictor for infection. ${ }^{4}$ Age at implantation, sex, etiology of the dilated cardiomyopathy, New York Heart Association class, conduction delay, hypertension, atrial fibrillation, diabetes, and obesity may also contribute to device infection. ${ }^{3,4,9}$ We considered all these variables when we analyzed the characteristics of the compared groups, using a logistic regression model for adjustment when significant differences were detected in order for the results to be as accurate as possible. Follow-up was similar to the one reported in other studies. ${ }^{5}$

Biventricular CIEDs implantation rate increased significantly after 2005 in Europe, but with large differences in absolute rates, the lowest being encountered in underdeveloped countries. ${ }^{2}$ These facts suggest that reuse of sterilized biventricular devices may have important clinical implications in these countries. Of great importance is the fact that a significant number of patients, from countries without full medical coverage, cannot receive device therapy when indicated, and also to the possible ways to resolve this problem. Some authors proved the feasibility of collecting CIEDs from crematories and/or funeral homes, finding a significant number of devices, both simple and biventricular, to be proper for reuse. ${ }^{15,16}$ Related to this aspect, in our study, we also describe a functional system consisting of receiving devices collected by a specialized institution, and reusing them after sterilization, that worked for 14 years now without problems.

Several studies described the safety of single-chamber and dual-chamber pacemakers' reuse, evaluating the complication rate in resterilized pacemakers compared with new ones. Baman et $\mathrm{al}^{5}$ performed a meta-analysis of 18 representative studies (from a total number of 32) conducted 
between 1970 and 2010, including a total number of 2,270 patients. They concluded that the differences in infectious complication rates were insignificant, but malfunction rate, although low, was higher for reused devices. Malfunctions in these studies were represented by connection system impairment, premature battery depletion, electromagnetic inhibition, spontaneous reprogramming, and inhibition by the pectoral muscle, and most of them could be attributed to electrical integrity impairment during explant and/or resterilization procedures. Most of these complications can now be avoided by according a special care for device explant procedures, device inspection, and interrogation before reusing. Kantharia et al ${ }^{17}$ published in 2012 a study including 53 patients implanted with resterilized simple pacemakers without finding an increase in infection rate, but the study has the drawback of lacking a control group. Also, Nava et al $^{3}$ performed a study including 603 patients implanted between 2007 and 2010, 307 with resterilized devices and 296 with new devices, showing the noninferiority of reused devices in terms of infection rate and device malfunction. These devices did have, though, lower life duration. Some authors describe this disadvantage of pacemaker reuse as a possible argument against this practice. ${ }^{18}$ There are little data in the literature regarding biventricular device reuse. To the best of our knowledge, one single study ${ }^{19}$ evaluated 81 patients receiving 106 biventricular defibrillators for CRT, concluding that reuse of these devices is safe, without a raise in complication rate (infection, defibrillator malfunction), but necessitating further studies for validation and confirmation. The study also lacked a control group. Evaluating specific aspects of defibrillator malfunction (eg, inappropriate shock delivery) was beyond the purpose of our study.

CIEDs' reuse was a routine about 20 years ago. In 1996, in Sweden, 5\% of the implant procedures used a resterilized device, without an obvious raise in infectious complications. Even the European Society of Cardiology and the American College of Cardiology/American Heart Association/North American Society of Pacing and Electrophysiology mentioned that device reuse should be considered and that it would contribute significantly to cardiac pacing cost-efficiency. Even so, this practice was abandoned in most of the developed countries because of responsibility issues, lack of precise safety proofs, and ethical issues., ${ }^{3,50-22}$ The current 2013 European Society of Cardiology guideline ${ }^{2}$ lacks any specifications in this direction. The ethical problems arise most from the uncertainty if proper resterilization can be accomplished after a first implantation and from the fact that devices labeled as for single use should undergo an approval process before reuse, if this action is intended. ${ }^{3,23}$ Legislation varies according to the country. For example, in France, CIEDs reuse is forbidden, but collecting them for donation is allowed. In Romania, as in other countries, there are no interdictions in the legislation regarding this issue. CIED reuse is practiced in many countries, and reporting the results is very important to clinical practice, in order to describe its safety. If CIEDs (simple/biventricular, pacemaker/defibrillator) reuse is proved safe, a more serious problem of ethics would be not to provide a sterilized device to a patient who needs it according to best current medical evidence, if a new device is not available and the patient lacks the possibility to acquire one. ${ }^{3}$

\section{Study limitations}

As we performed a retrospective study from patient files and procedural logbooks, we lacked certain types of data that would have made the analysis more accurate, like chronic pacing and sensing thresholds, functioning modality, and functioning percent. We partly reduced this drawback by acquiring the acute pacing and sensing threshold values, and by the fact that steps had been taken in order to insure the highest percentage of biventricular pacing possible, in all devices.

The number of patients is limited by the fact that this is a single-center study and CRT interventions are significantly less frequent than implantations of single- and dual-chamber pacemakers and/or defibrillators.

\section{Conclusion}

Reuse of biventricular devices seems safe in terms of infectious complications, early battery depletion, and generator malfunction compared to new devices, both in general population and in the elderly population. This seems to be a promising alternative for patients needing CRT when new devices are not available and cannot be procured in a safe period of time. Future studies are needed in order to strengthen these conclusions.

\section{Acknowledgment}

This paper was published under the frame of European Social Found, Human Resources Development Operational Programme 2007-2013, project number POSDRU/159/1.5/S/136893.

\section{Disclosure}

The authors report no conflicts of interest in this work. 


\section{References}

1. Ghio S, Freemantle N, Scelsi L, et al. Long term left ventricular reverse remodeling with cardiac resynchronization therapy: results from the CARE-HF trial. Eur Heart J. 2009;22:480-488.

2. Brignole M, Auricchio A, Baron-Esquivias G, et al. 2013 ESC guidelines on cardiac pacing and cardiac resynchronization therapy. The task force on cardiac pacing and resynchronization therapy of the European Society of Cardiology (ESC). Developed in collaboration with the European Heart Rhythm Association (EHRA). Eur Heart J. 2013;34:2281-2329.

3. Nava S, Morales JL, Márquez MF, et al. Reuse of pacemakers: comparison of short and long-term performance. Circulation. 2013;127(11): $1177-1183$.

4. Margey R, McCann H, Blake G, et al. Contemporary management of and outcomes from cardiac device related infections. Europace. 2010; 12:64-70.

5. Baman TS, Meier P, Romero J, et al. Safety of pacemaker reuse: a meta-analysis with implications for underserved nations. Circ Arrhythm Electrophysiol. 2011;4(3):318-323.

6. Kautzner J, Riedlbauchova L, Cihak R, Bytesnik J, Vancura V. Technical aspects of implantation of LV lead for cardiac resynchronization therapy in chronic heart failure. Pacing Clin Electrophysiol. 2004; 27:783-790.

7. VanArtsdalen J, Dorr Goold S, Kirkpatrick JN, Goldman E, Eagle K, Crawford T. Pacemaker reuse for patients in resource poor countries: is something always better than nothing? Prog Cardiovasc Dis. 2012;55: 300-306.

8. Linde CL, Bocray A, Jonsson H, Rosenqvist M, Rådegran K, Rydén L. Re-used pacemakers-as safe as new? A retrospective case-control study. Eur Heart J. 1998;19(1):154-157.

9. Cengiz M, Okutucu S, Ascioglu S, et al. Permanent pacemaker and implantable cardioverter defibrillator infections: seven years of diagnostic and therapeutic experience of a single center. Clin Cardiol. 2010; 33(7):406-411.

10. Dickstein K, Vardas PE, Auricchio A, et al; ESC Committee for Practice Guidelines (CPG). 2010 Focused update of ESC guidelines on device therapy in heart failure. An update of the 2008 ESC guidelines for the diagnosis and treatment of acute and chronic heart failure and the 2007 ESC guidelines for cardiac and resynchronization therapy. Developed with the special contribution of the Heart Failure Association and the European Heart Rhythm Association. Eur Heart J. 2010;31:2677-2687.

11. Vardas PE, Auricchio A, Blanc JJ, et al; European Society of Cardiology; European Heart Rhythm Association. Guidelines for cardiac pacing and cardiac resynchronization therapy: the task force for cardiac pacing and cardiac resynchronization therapy of the European Society of Cardiology. Developed in collaboration with the European Heart Rhythm Association. Eur Heart J. 2007;28:2256-2295.
12. Gregoratos G, Abrams J, Epstein AE, et al; American College of Cardiology/American Heart Association Task Force on Practice Guidelines/North American Society for Pacing and Electrophysiology Committee to Update the 1998 Pacemaker Guidelines. ACC/AHA/ NASPE 2002 Guideline update for implantation of cardiac pacemakers and antiarrhythmia devices: summary article. A report of the American College of Cardiology/American Heart Association Task Force on Practice Guidelines (ACC/AHA/NASPE Committee to Update the 1998 Pacemaker Guidelines). Circulation. 2002;106:2145-2161.

13. Ellenbogen KA, Kay GN, Lau CP, Wilkoff BL. Clinical Cardiac Pacing, Defibrillation, and Resynchronization Therapy. 4th ed. Philadelphia: Elsevier Saunders; 2011.

14. Sohail MR, Uslan DZ, Khan AH, et al. Risk factor analysis of permanent pacemaker infection. Clin Infect Dis. 2007;45:166-173.

15. Baman TS, Crawford T, Sovitch P, et al. Feasibility of postmortem device acquisition for potential reuse in underserved nations. Heart Rhythm. 2012;9(2):211-214.

16. Zamani P, Kirkpatrick JN, Litzky LA, Verdino RJ. Longevity of implantable electrophysiology devices explanted from patients having autopsy in hospitals. Am J Cardiol. 2012;110(11):1643-1645.

17. Kantharia BK, Patel SS, Kulkarni G, et al. Reuse of explanted permanent pacemakers donated by funeral homes. Am J Cardiol. 2012; 109(2):238-240.

18. Baman TS, Kirkpatrick JN, Romero J, et al. Pacemaker reuse: an initiative to alleviate the burden of symptomatic bradyarrhythmia. Circulation. 2010;122:1649-1656.

19. Pavri BB, Lokhandwala Y, Kulkarni GV, Shah M, Kantharia BK, Mascarenhas DA. Reuse of explanted, resterilized implantable cardioverter-defibrillators: a cohort study. Ann Intern Med. 2012;157(8): 542-548.

20. Hailey D, Jacobs PD, Ries NM, Polisena J. Reuse of single use medical devices in Canada: clinical and economic outcomes, legal and ethical issues, and current hospital practice. Int J Technol Assess Health Care. 2008;24:430-436.

21. Kirkpatrick JN, Papini C, Baman TS, et al. Reuse of pacemakers and defibrillators in developing countries: logistical, legal, and ethical barriers and solutions. Heart Rhythm. 2010;7:1623-1627.

22. Aragam KG, Baman TS, Kirkpatrick JN, et al. The ethics of pacemaker reuse: might the best be the enemy of the good? Heart. 2011;97: 2005-2006.

23. Rydén L. Re-use of devices in cardiology: proceedings from a Policy Conference at the European Heart House, 5-6 February, 1998. Eur Heart J. 1998;19:1628-1631.
Clinical Interventions in Aging

\section{Publish your work in this journal}

Clinical Interventions in Aging is an international, peer-reviewed journal focusing on evidence-based reports on the value or lack thereof of treatments intended to prevent or delay the onset of maladaptive correlates of aging in human beings. This journal is indexed on PubMed Central, MedLine,

\section{Dovepress}

CAS, Scopus and the Elsevier Bibliographic databases. The manuscript management system is completely online and includes a very quick and fair peer-review system, which is all easy to use. Visit http://www.dovepress. com/testimonials.php to read real quotes from published authors. 